\title{
Stability Analysis of GNSS Control Point Network for Material Displacement Monitoring on the Slopes using Stability Monument Evaluation and Adjustment Data Processing Scheme: Preliminary Result
}

\author{
Vera Sadarviana*, Brian Bramanto, Teguh P. Sidiq, Mohamad Gamal, Daniel A. Tampubolon, Rahmadi Hilmafizar, \\ Reyhan A. Biantoro, Hana Alifiyanti., Muhammad T. A. Faruq, Muhammad F Muttaqin \\ Geodesy Research Group, Insitut Teknologi Bandung, Indonesia
}

\begin{abstract}
The Global Navigation Satellite System (GNSS) has been used widely for hazards monitoring, such as landslide or material displacement on the slope due to its high accuracy and precision positioning. However, to assure its accuracy and precision, a further data quality and site assessment must be taken into account. In such a way, it is possible to determine whether the site monitoring is moved or not. Six location of GNSS observation points were established based on the geological structure and the terrain slopes. Satellite visibilities analysis, multipath analysis, and kinematic precise point positioning analysis were performed to assess the GNSS data quality and the monitoring stability. These procedures will determined the further processing scheme for each site monitoring. Some of areas experience the indication of cracks in road and building construction, which lead into an assumption of the displacement has been accumulated in a sub meter fraction. Thus, accounting all of those aspects, first adjustment data processing was implemented to achieve the preliminary results of the first observation.
\end{abstract}

\section{Introduction}

The Toll road is intended to shorten travel time with short and congestion-free routes. Hence, many Toll road routes pass through hills or mountains and create new slopes. Toll road vehicles are traversed by various types and weights of vehicles. The ability of vehicles to travel on toll roads is not the same because there are vehicles that carry goods with the maximum weight unable to pass the toll road with a minimum speed of $60 \mathrm{~km} /$ hour. This situation causes vibration to the morphology of the surrounding area, including the slope. This makes the slope susceptive to landslides because there is vibration as a trigger.

The Cipularang toll road connecting Bandung and Jakarta have high vehicle density and cause congestion. These conditions give the vibrations continuously and repeatedly. Another trigger factor is rain. As a result, the Cipularang toll road experiences avalanches in several segments like $\mathrm{km} 92$ and $\mathrm{km} 96$. For mitigation, the stability of the slope material monitoring that is by putting up points on the slopes and conduct periodic position observation.

Geologically, some areas of the Cipularang toll road have clay rocks that are hard, easy to swell-shrink if it is affected by rain and heat. In addition, the physical condition of the road in the form of fill the embankment makes the toll road area easy to move. So many factors make the Cipularang toll road area vulnerable to movement. Each points monitor can move or change its position due to the influence of one or a combination of these factors.

Monitoring of ground movements is defined by the difference between two or more position observation periodic. The stability of the monitoring point used must be ascertained first so as not to misunderstand the existing position changes. On the other hand, the quality of the observation method must be ensured so that the resulting differences do not come from errors or inconsistencies of observation. In this paper, the GNSS data were first assess to ensure the quality of the data, then some proccessing scheme was accounted to estimate the first network solution as a preliminary results.

\section{Data and Method}

Gunung Hedjo, West Java, Indonesia is traversed by Bandung - Jakarta highly occupied toll road, however, Gunung Hedjo suffers from numerous landslides. Thus, the high accuracy and precision positioning monitoring in this area is indeed needed. Six GNSS observation sites were established to monitor the slopes stability on the research area.

Corresponding author: vsadarviana@ gmail.com 


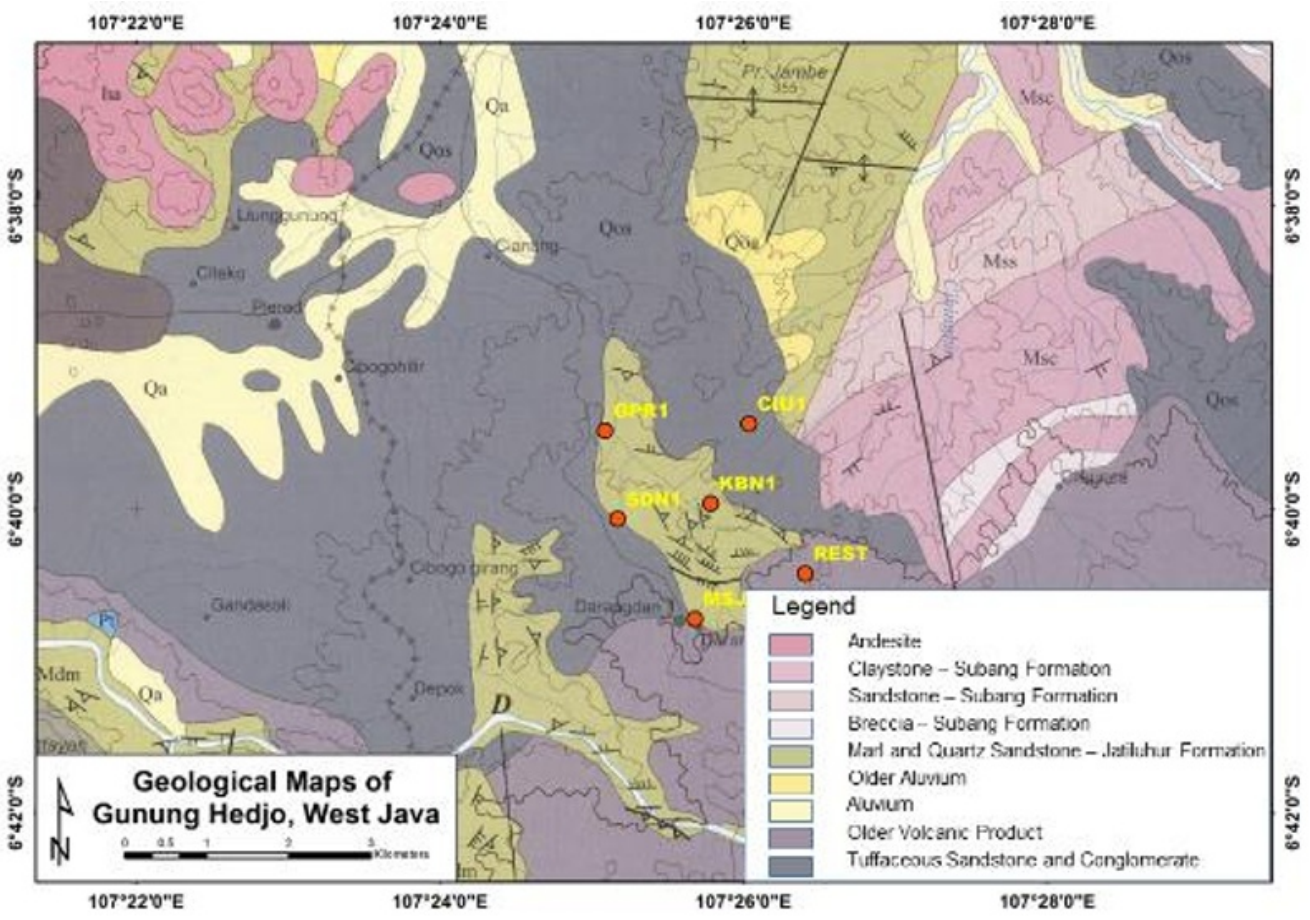

Fig. 1 Observation monitoring sites (Orange Dot) overlaid with the geological map

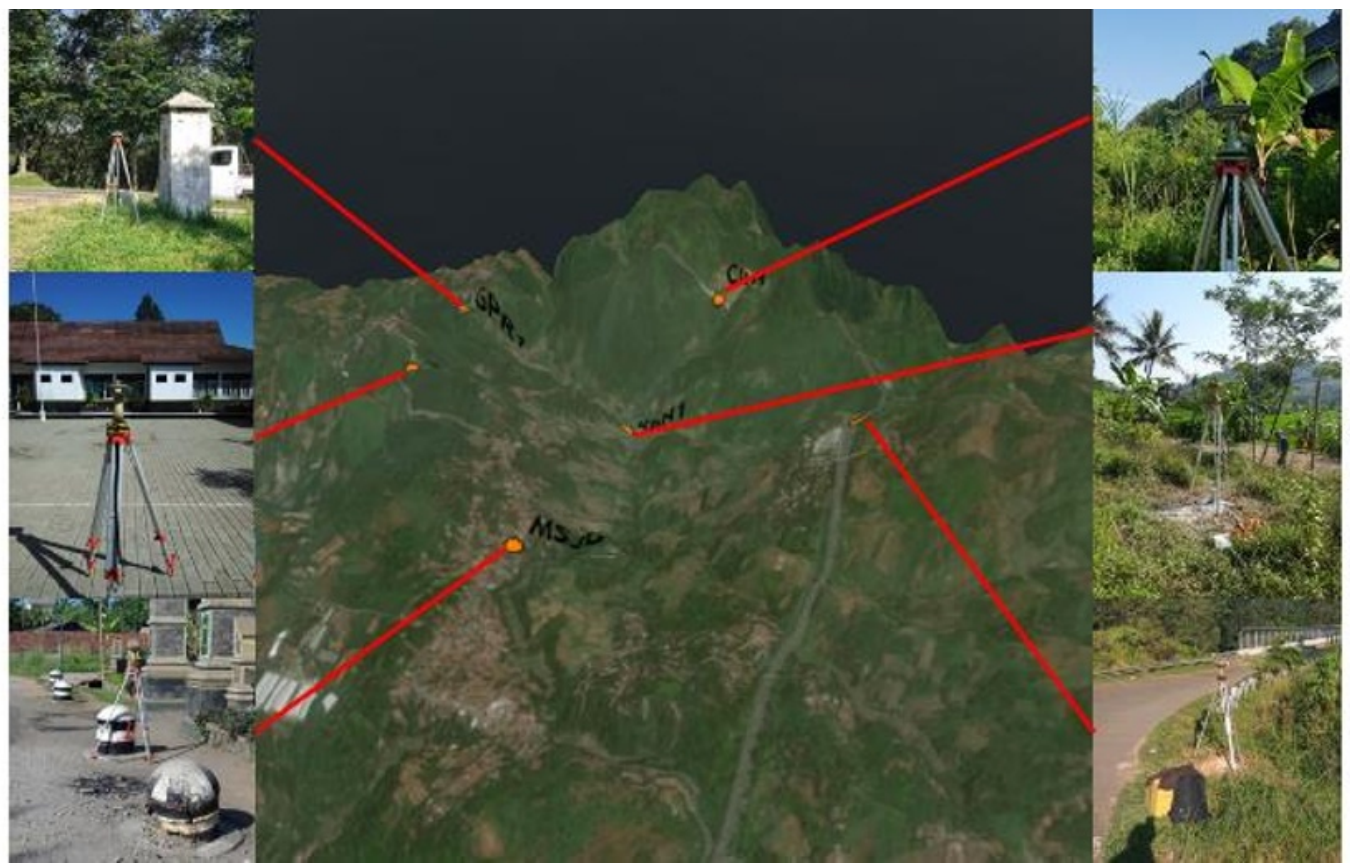

Fig. 2 3D view of observation site (orange dot) and the condition of measurement

The geological structure and the terrain slope were considered for the site monitoring placement. Fig. 1 shows the geological structure over the research area. It could be seen that the area are mostly consists of quaternary and Miocene rock. Tuffaceous sandstone, conglomerate and alluvium are commonly found on the quaternary rock, while marl, quartz sandstone and volcanic product are commonly found on the Miocene rock. The landslide are often occurred on the tuffaceous sandstone and other volcanic product [1, 2, 3]. Fig. 2 indicates the terrain over the research area. The elevation height of the research area is vary from 350 to 550 meter above sea level.

The classical geodetic campaign was carried out on July 2018 using four Topcon GR-3 GNSS receiver, Leica GS-08 and Leica GRX-1200. In the first campaign, 10 baseline observation with duration of 3 hours each and 1 baseline observation with duration of 6 hours had been held out. All of the observation were collected in $1 \mathrm{~Hz}$ sampling interval. One Continuous Operating Reference System (CORS) GNSS station used as a reference, it was assumed that the CORS GNSS is not affected from any 
mass displacement. The CORS GNSS is located for about 30 kilometers away in Bandung, West Java, Indonesia.

Before the differencing and network adjustment process is carried out, the data quality assessment must be done. The data quality assessment is consists of satellite visibilities analysis, multipath analysis, Signal to Noise Ratio (SNR) analysis and Kinematic Precise Point Positioning (KPPP) analysis.

Satellite visibilities is one way to estimate the precision of the estimated position which correlated with the geometry of the observed satellite, namely Dilution of Precision (DOP). DOP can be divided into several terms which are correlated by the position or time, such as vertical DOP (VDOP), horizontal DOP (HDOP), position DOP (PDOP) and time DOP (TDOP). Those terms can be generalize by using Geometric DOP (GDOP) term. DOP can be described as [4]:

$$
\begin{aligned}
& G D O P=\frac{\sqrt{\sigma_{x_{u}}^{2}+\sigma_{y_{u}}^{2}+\sigma_{z_{u}}^{2}+\sigma_{c t b}^{2}}}{\sigma_{U E R E}} \\
& \sqrt{\sigma_{x_{u}}^{2}+\sigma_{y_{u}}^{2}+\sigma_{z_{u}}^{2}}=P D O P . \sigma_{U E R E} \\
& \sqrt{\sigma_{x_{u}}^{2}+\sigma_{y_{u}}^{2}}=H D O P . \sigma_{U E R E} \\
& \sigma_{z_{u}}=\text { VDOP. } \sigma_{U E R E} \\
& \sigma_{c t_{b}}=\text { TDOP. } \sigma_{U E R E}
\end{aligned}
$$

where $\sigma_{x}, \sigma_{y}, \sigma_{z}$ is the standard deviation of the threedimensional position and $\sigma_{c t b}$ is the standard deviation of the clock timing defined in distance at the specified location $(u)$.

Multipath is one of the unavoidable source of error in GNSS signal propagation [5]. Although it is difficult to model the error, it is very necessary for us to describe the quality of the monitoring site. The multipath can be investigate by applied the Multipath Combination (MPC) algorithm. The MPC can be expressed as [6]:

$$
\begin{aligned}
& M P 1=P_{1}-\frac{f_{1}^{2}+f_{2}^{2}}{f_{1}^{2}-f_{2}^{2}} L_{1}+\frac{2 f_{2}^{2}}{f_{1}^{2}-f_{2}^{2}} L_{2} \\
& M P 2=P_{2}-\frac{2 f_{2}^{2}}{f_{1}^{2}-f_{2}^{2}} L_{1}+\frac{f_{1}^{2}+f_{2}^{2}}{f_{1}^{2}-f_{2}^{2}} L_{2}
\end{aligned}
$$

where $P$ and $L$ are the GNSS pseudorange and carrier phase range respectively and $f$ is the frequency of the carrier phase. The first order of ionospheric delay and geometric range could be eliminated by using these linear combination.

In general, KPPP refers as an absolute point positioning which adopt the linear combination and other supporting product to reduce and eliminate the huge amount of error in the GNSS signal propagation. KPPP uses ionospheric-free linear combination to eliminate the first order ionospheric error and precise orbit and clock to reduce the orbital and satellite clock error. The linear combination of KPPP can be described on the following equation $[7,8]$ :

$P_{I F}=\frac{f_{1}^{2} P_{2}-f_{2}^{2} P_{2}}{f_{1}^{2}-f_{2}^{2}}$
$L_{I F}=\frac{f_{1}^{2} L_{2}-f_{2}^{2} L_{2}}{f_{1}^{2}-f_{2}^{2}}$

Thus, those observation can be used to determine the unknown parameter. The unknown parameter in the KPPP method include three coordinate parameter $(X, Y, Z)$, a receiver clock bias $(d t)$, a wet zenith tropospheric delay and float ambiguity.

Considering all of the previous pre-process analysis, double difference (DD) positioning applied to obtain the final baseline solution. DD conducted by differencing two single difference (SD) observation. The phaserange SD between receivers can be described as follows [9]:

$$
\begin{gathered}
\Delta L_{A B}^{j}=\Delta \rho_{A B}^{j}+d_{\text {trop } A B}^{j}-d_{i o n A B}^{j}+c(d t-d T)_{A B}^{j} \\
+M L_{A B}^{j}+\lambda N_{A B}^{j}+\delta L_{A B}^{J}
\end{gathered}
$$

Where $\Delta$ is the difference between receivers $\mathrm{A}$ and $\mathrm{B}$. $\rho$ denotes the geometric range. $d_{\text {trop } A B}^{j}$ and $d_{\text {ion } A B}^{j}$ denote the delay of tropospheric and ionopsheric respectively. $C$ denotes the speed of light while $d T$ refers to receiver clock bias. The remaining $M L, \lambda N$ and $\vartheta L$ refer to multipath, integer ambiguity and noise. The superscript $-j$ corresponds with the observed satellite. Thus, by subtracting two SD observation, DD can be defined as follows:

$\Delta \nabla L_{A B}^{j k}=\Delta \rho_{A B}^{j k}+M L_{A B}^{j k}+\lambda N_{A B}^{j k}+\delta L_{A B}^{J k}$

Where the superscript- $j$ and $k$ define the two observed satellite on the same epoch. The shorter baseline might eliminated the atmospheric bias.

\section{Data Observation Assessment}

\subsection{Satellite Visibilities Analysis}

Table 1 shows that loops formed from 3 monitor points produce a horizontal closure in easting and northing axis, a vertical closure in height element. The biggest loop closure occurs in loops 2 and 5 . It directs suspicion towards the points of GPR1 and KBN1.

Selected satellite visibilities shown in Fig. 3 and Fig. 4 for GPR1 and KBN1 site respectively. GPR1 and KBN1 were chosen to emphasize the two different surrounding. GPR1 uses Leica GS08 while KBN1 uses Topcon GR3 GNSS Receiver. GPR1 located near the highway while KBN1 located in the middle of cropland as illustrated in 
Fig. 5. Cycle slips then detected by the jump of observed phaserange in the data observation. GPR1 suffers a lot of cycle slip which occurred in the direction of about $30^{\circ}$, $150^{\circ}, 240^{\circ}-300^{\circ}$ and $330^{\circ}$ from North with the low satellite elevation as indicated by the red line. Contrarily, KBN1 experiences no cycle slip for all of the observation. Closely look at the surrounding of GPR 1 , the cycle slips in the direction of $30^{\circ}, 150^{\circ}$ and $240^{\circ}-300^{\circ}$ are likely due to the obstructed trees, while in the direction of $330^{\circ}$ is due to the existence of the pillar. The occurrence of cycle slips will decrease the successful rate of resolved ambiguity. Further data treatment should be applied to minimize the unresolved ambiguities, such as, repair all cycle-slips or remove the cycle slips from the observed data.

Although the data collected on the same period of the observation and the distance between each site were relatively close, the number of observed satellites might be different. Fig. 6 shows the number of observed satellite as well as the DOP. As seen on Fig. 6, the variation of number of satellite in GPR1 is slightly larger than in KBN1. The statistic over those sites indicate that the mean number of observed satellite for GPR1 and KBN1 are 17 and 18 satellite respectively, while the standard deviation are 2 and 1 satellites for each site. As mentioned before, high disturbed surrounding at the low elevation satellite. This condition interferes the GNSS signal propagation.

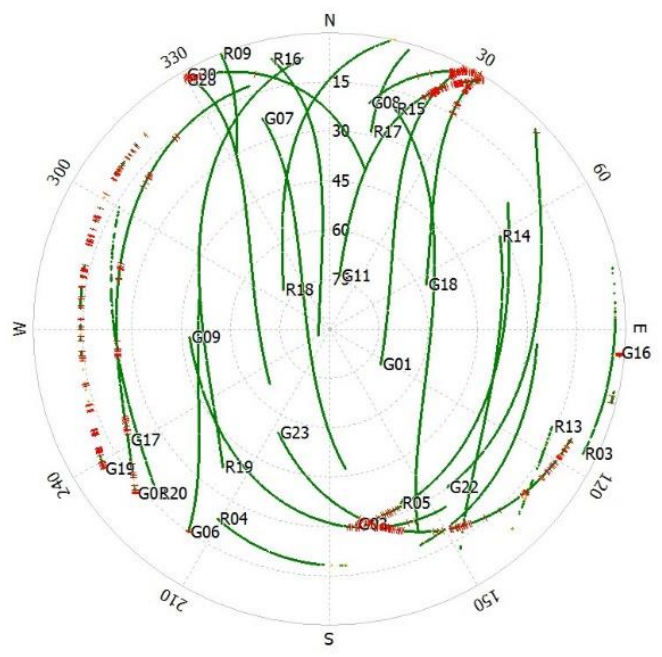

Fig. 3 The skyplot of satellite over GPR1 monitoring sites. Red lines indicate the occurred cycle slip on the observed data

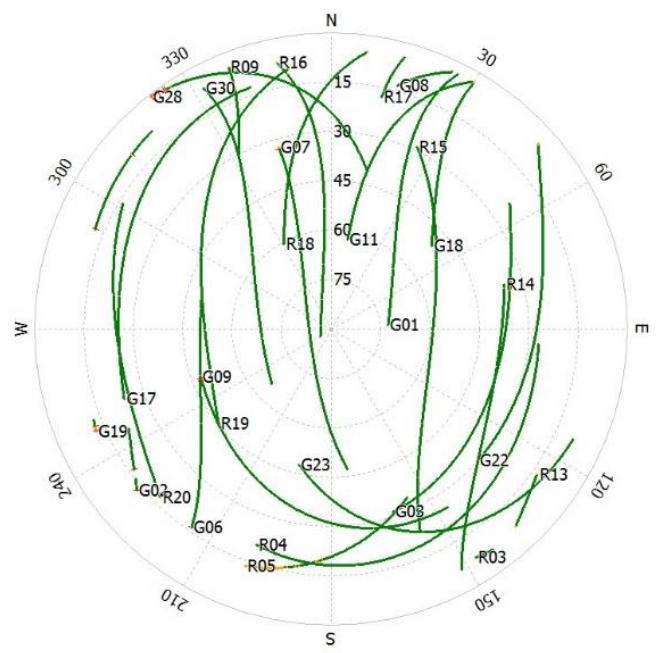

Fig. 4 The skyplot of satellite over KBN1 monitoring sites. Red lines indicate the occurred cycle slip on the observed data

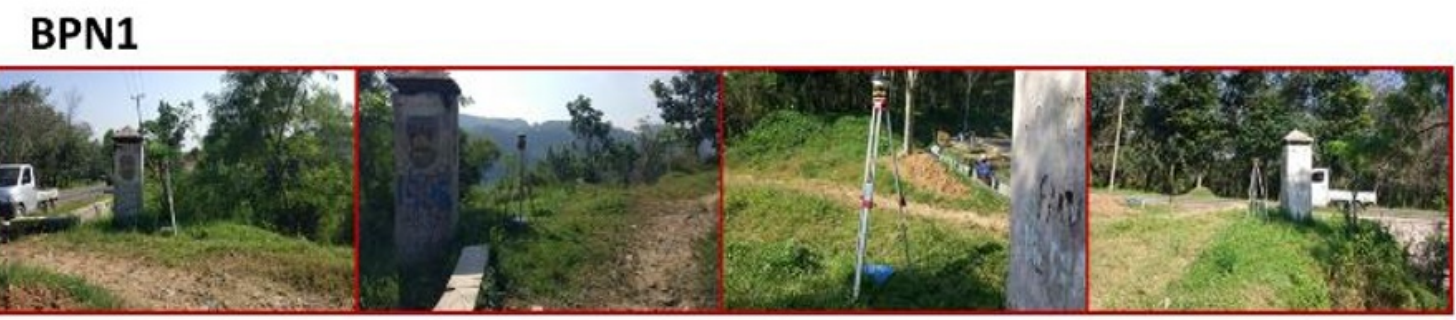

\section{SAWH}

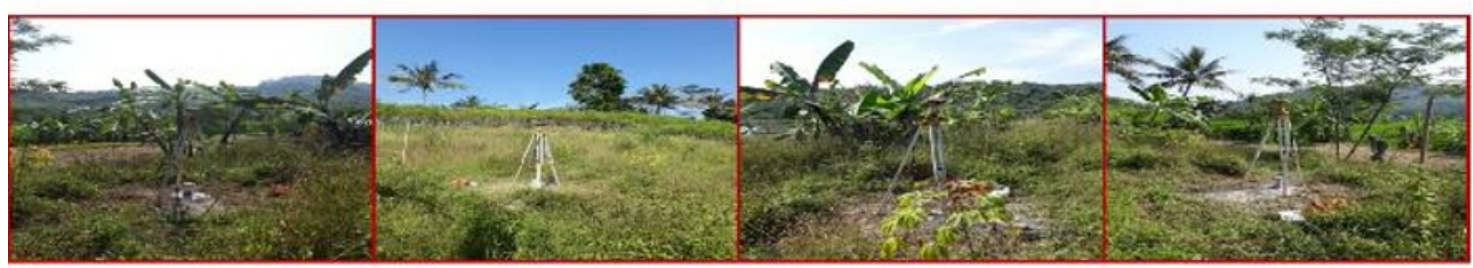

Fig. 5 Surrounding of selected sites. GPR1 shown on the top of figure, while KBN1 shown on the bottom of figure. Left to the right picture indicate the North, East, South and West orientation respectively 

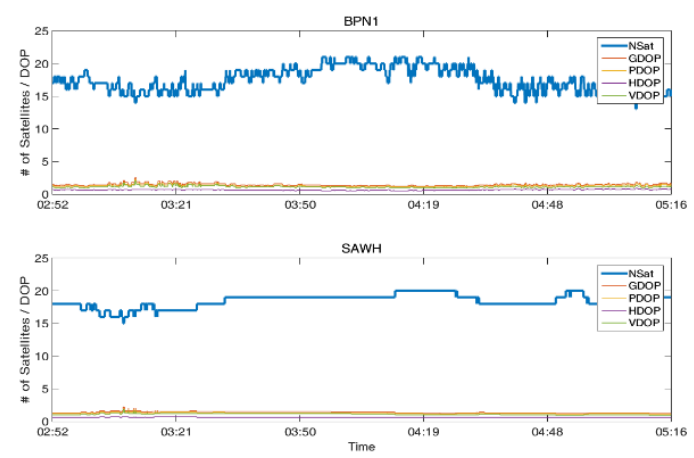

Fig. 6 Number of observed satellite and DOP for each site. GPR1 shown on the top of figure, while KBN1 shown on the bottom of figure

\subsection{Multipath Analysis}

Multipath is a localized effect, which depends on the local surrounding site. Fig. 7 illustrates the multipath signals. Any range measurement will severely disturbed by the multipath effect, due to the longer propagation path from the satellite to receiver.

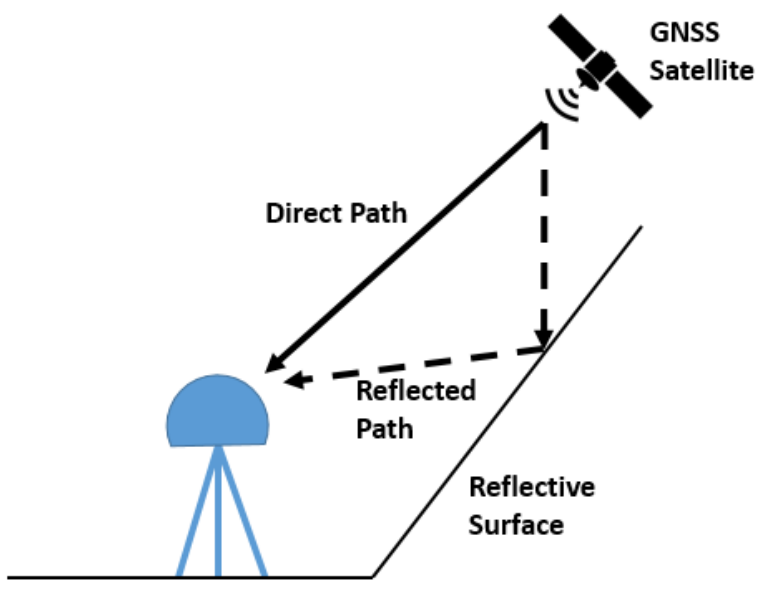

Fig. 7 Direct path and reflected path/multipath received signal

Fig. 8 and Fig. 9 show the L1 and L2 multipath for each site. In general, the multipath error variation in KBN1 relatively larger than those in GPR1. This is likely due to the typical noise from the receiver itself. All of the multipath error estimated from Topcon GR-3 show the similar pattern and variation. However, further investigation for the multipath error show that the multipath error variation tends to be more random and have significant multipath error.

Fig. 10 until Fig. 13 show the L1 and L2 skyplot of multipath for each site. Multipath usually occurred on the low elevation satellite angle, however, the significant multipath error occurred on the high elevation satellite angle on GPR1. The significant multipath error mostly occurred in the direction of about 330 degrees from North. Due to the close distant between pillar and the antenna, multipath significant error might be occurred even in the high elevation satellite angle. Differ from GPR1, multipath error which occurred in KBN1 relatively constant on all of the observation and tend to decrease on the high elevation satellite. 


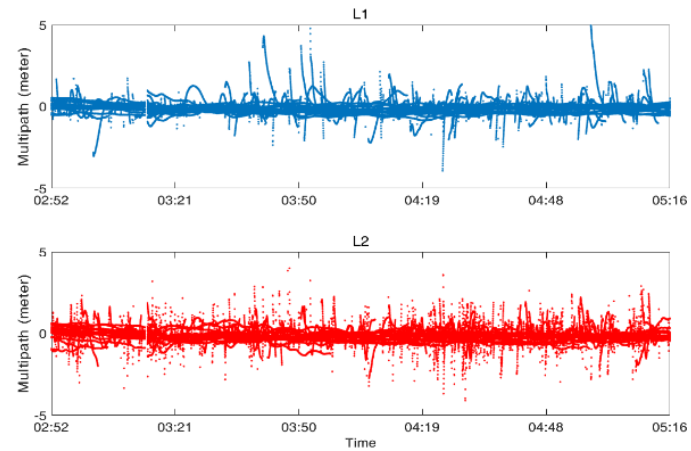

Fig. 8 Multipath error on GPR1
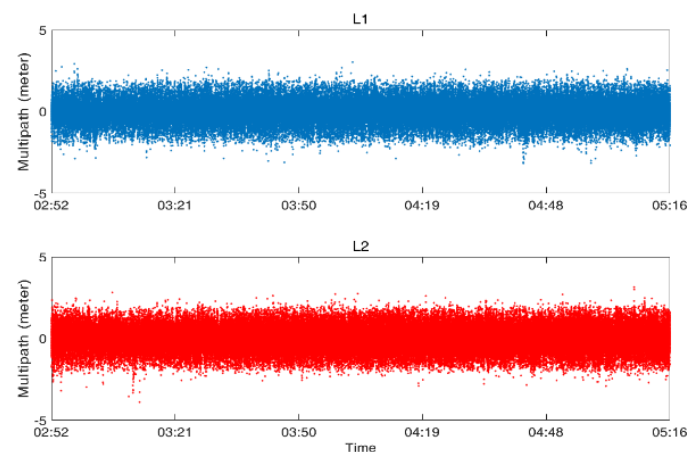

Fig. 9 Multipath error on KBN1

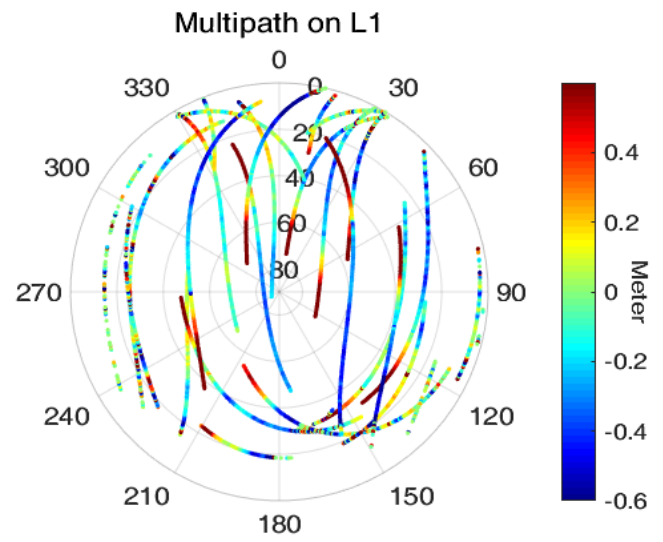

Fig. 10 Skyplot multipath L1 on GPR1

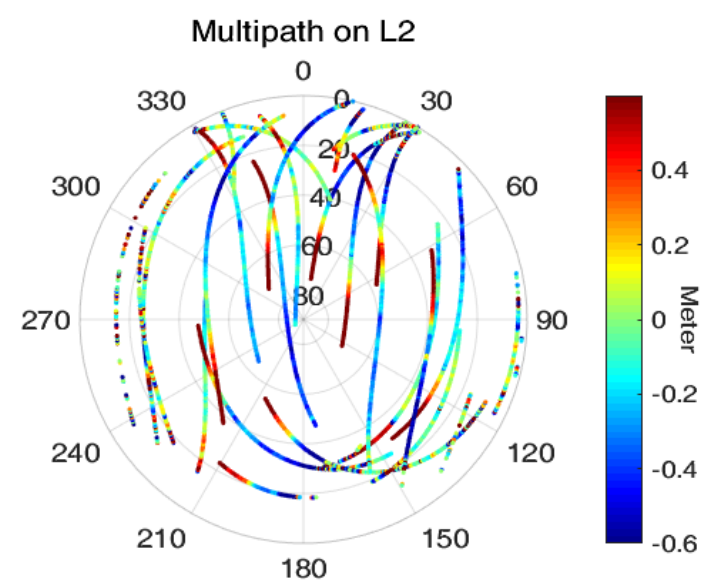

Fig. 11 Skyplot multipath L2 on GPR1 


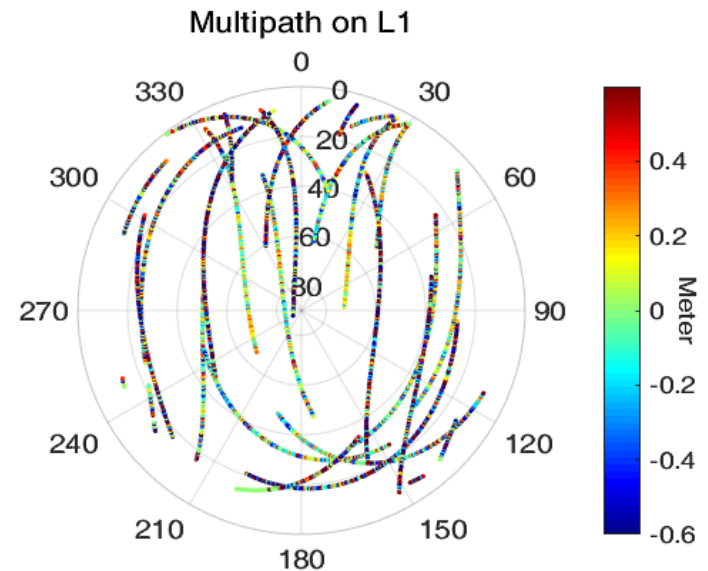

Fig. 12 Skyplot multipath L1 on KBN1

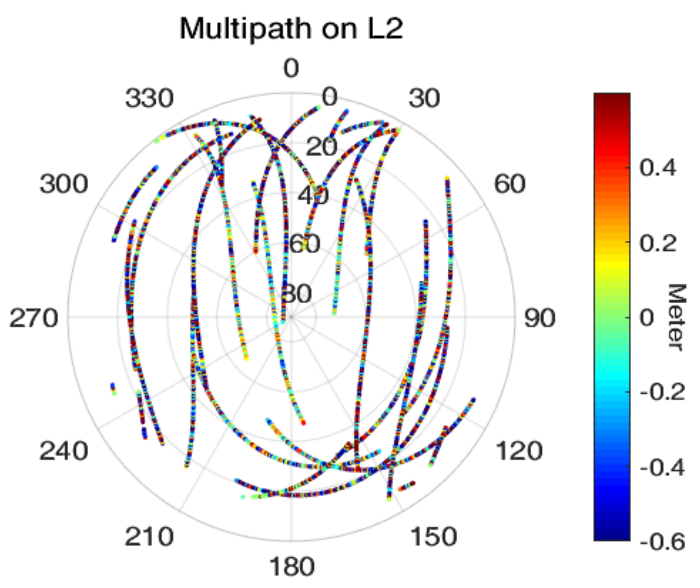

Fig. 13 Skyplot multipath L2 on KBN1

\subsection{Kinematic Precise Point Positioning Analysis}

KPPP was conducted to analyze the effect of observed geometry satellite and multipath. IGS final orbit and clock correction was used to eliminate the orbit and satellite clock bias. LAMBDA ambiguity resolution search was used to estimate the carrier phase ambiguities with $0^{\circ}$ of elevation mask angle. Final solution used Forward and Backward filter.

Fig. 14 until Fig. 17 show the estimated position in ENU coordinate system for GPR1 and KBN1. Due to high obstructed surrounding, GPR1 estimated position tend to spread within $10 \mathrm{~cm}$, while KBN1 only half of it. A tendency towards the northeast-southwest direction is indicated in GPR1, this is as a result of the reflector surface (pillar) in the direction of about $330^{\circ}$. The receiver may receive both of the direct and the reflected signal from GNSS satellites [10].
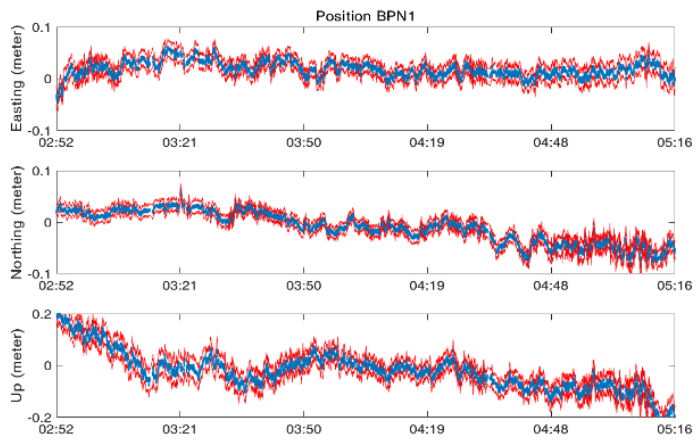

Fig. 14 GPR1 estimated position (ENU coordinate system). Blue dot and red line refer to estimated positon and its standard deviation 


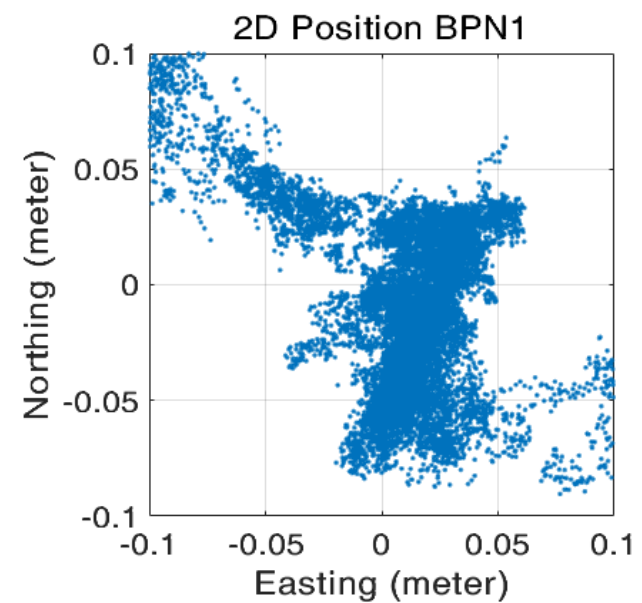

Fig. 15 GPR1 2D position
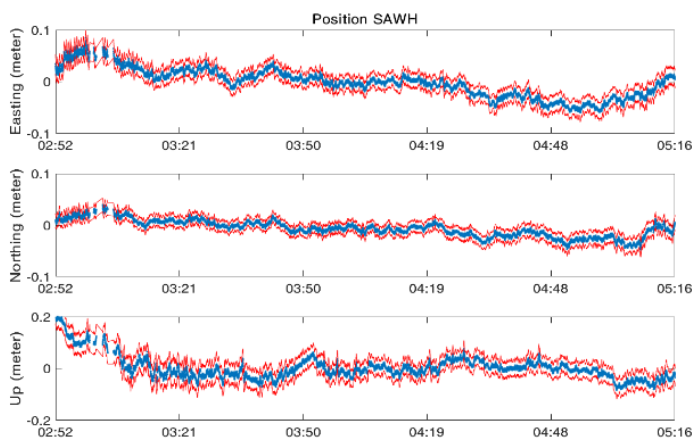

Fig. $16 \mathrm{KBN} 1$ estimated position (ENU coordinate system). Blue dot and red line refer to estimated positon and its standard deviation

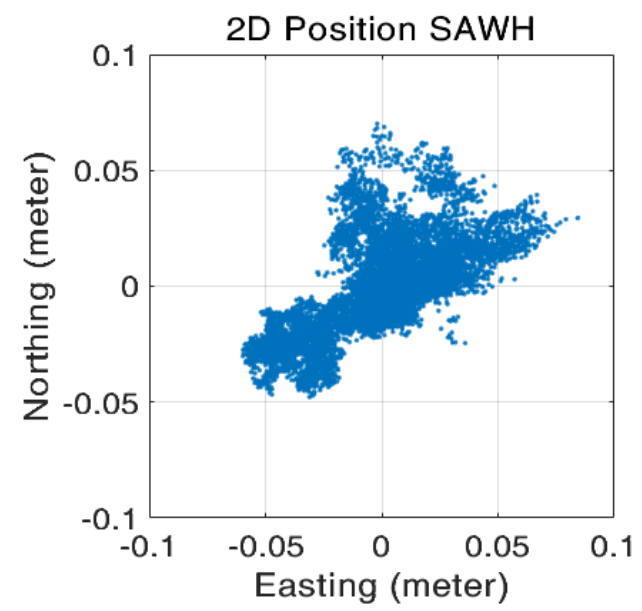

Fig. 17 KBN1 2D position

\section{Network Adjustment}

Accounting all of those aspects, first preliminary network adjustment is conducted. Baseline processing is done by using Leica Geo Office 8.1. The intermitted data is first removed from the observation data. Furthermore, $20^{\circ}$ elevation mask angle was applied to minimize the multipath effect from low elevation satellite.

Five loops were formed for network adjustment. Loop closure are within mm level for horizontal and $\mathrm{cm}$ level for vertical. Further analysis revealed that loop 1, 2 and 5 slightly worse than loop 3 and 4 for vertical closure. Site condition on GPR1 and CIUJ were relatively worse than the others. Both of them were one of the observed point in loop 1, 2 and 3. Thus final network adjustment is applied.

Table 2 and Table 3 summarized the final solution.

Table 1 Loop closure for each formed loops (unit: meter)

\begin{tabular}{lllll} 
Loop & From & To & Easting & Northing Height \\
\hline
\end{tabular}




\begin{tabular}{|c|c|c|c|c|c|}
\hline \multirow{3}{*}{1} & KBN1 & RA97 & \multirow{3}{*}{-0.0012} & \multirow{3}{*}{0.0036} & \multirow{3}{*}{-0.0130} \\
\hline & RA97 & CIUJ & & & \\
\hline & CIUJ & KBN1 & & & \\
\hline \multirow{3}{*}{2} & KBN1 & SDN1 & \multirow{3}{*}{-0.0055} & \multirow{3}{*}{0.0021} & \multirow{3}{*}{0.0172} \\
\hline & SDN1 & GPR1 & & & \\
\hline & GPR1 & KBN1 & & & \\
\hline \multirow[t]{2}{*}{3} & KBN1 & SDN1 & \multirow{2}{*}{-0.0082} & \multirow{2}{*}{0.0009} & \multirow{2}{*}{0.0072} \\
\hline & SDN1 & MSJD & & & \\
\hline
\end{tabular}

\begin{tabular}{clllll}
\multicolumn{1}{c}{ MSJD } & KBN1 & & & \\
\hline & MSJD & KBN1 & & & \\
& KBN1 & RA97 & -0.0006 & -0.0019 & -0.0060 \\
& RA97 & MSJD & & & \\
\hline \multirow{4}{*}{5} & CIUJ & KBN1 & & & \\
& KBN1 & GPR1 & -0.0022 & 0.0003 & -0.0170 \\
& GPR1 & CIUJ & & & \\
\hline
\end{tabular}

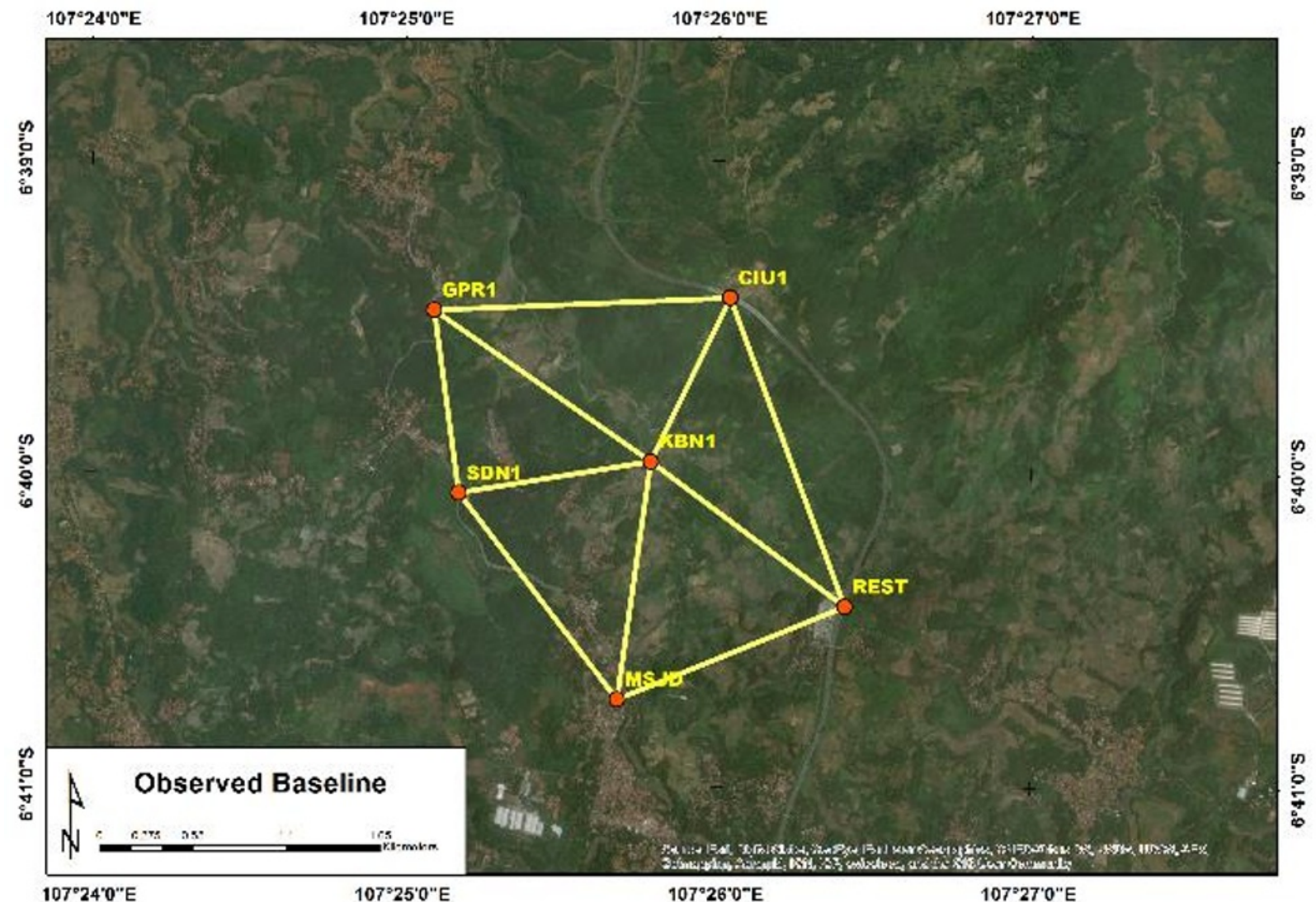

Fig. 18 Formed baseline indicated as yellow lines

Table 2 Adjusted point coordinates in UTM 48S (unit: meter)

\begin{tabular}{llll} 
Point & Easting & Northing & Height \\
\hline \hline GPR1 & 767386.1438 & 9263276.7253 & 437.1725 \\
CIUJ & 768999.8599 & 9263484.8570 & 455.5000 \\
MSJD & 768439.6020 & 9261119.1939 & 511.8749 \\
RA97 & 769772.5571 & 9261416.1212 & 570.2214 \\
KBN1 & 768651.2936 & 9262489.8261 & 401.3860 \\
SDN1 & 767551.8261 & 9262292.4662 & 490.6122
\end{tabular}

Table 3 Standard deviation (unit: meter)

\begin{tabular}{lrrr} 
Point & \multicolumn{1}{c}{ Sd. E } & \multicolumn{1}{c}{ Sd. N } & \multicolumn{1}{l}{ Sd. H } \\
\hline \hline GPR1 & 0.0016 & 0.0015 & 0.0044 \\
CIUJ & 0.0001 & 0.0001 & 0.0003
\end{tabular}

$\begin{array}{llll}\text { MSJD } & 0.0017 & 0.0015 & 0.0043 \\ \text { RA97 } & 0.0015 & 0.0013 & 0.0038 \\ \text { KBN1 } & 0.0015 & 0.0013 & 0.0038 \\ \text { SDN1 } & 0.0018 & 0.0016 & 0.0046\end{array}$

\section{Conclusion}

The acquisition of reliable landslide monitoring points needs to be done by reducing the lack of observations. Observations using different types and brands must be considered the adjustment process based on the characteristics of the device. The location of GPR1 which is on the side of the road and beside the area of plantation makes more cycleslip, the recorded number of satellite changes rapidly more than $\mathrm{KBN} 1$ in the middle of the cropland, but KBN1 has a large multipath error. The result of the estimated position spreading of $\mathrm{KBN} 1$ is smaller than GPR1. Temperature at observation will cause a 
refractive effect and reduce the level of accuracy of the observation data, especially for points that are on the edge of the highway. For the purpose, several steps must be taken towards the results of observational data, such as an elevation mask angle of at least $15^{\circ}$ to reduce errors due to multipath, choose satellite data with a high SNR value or remove satellite data that has many cycle slips.

\section{References}

1. Busthan, A. M. Imran, L. Samang, and M. Ramli. (2016) Engineering Geological Study of MalinoManipi Landslide Susceptibility South Sulawesi Indonesia. APRN Journal of Engineering and Applied Sciences, 11(15).

2. Hiromitsu Yamagishi and Yoji Ito (1994) Relationship of the landslide distribution to geology in Hokkaido, Japan. Engineering Geology 38, 189203.

3. Masahiro Chigira (2002) Geologic factors contributing to landslide generation in a pyroclastic area: August 1998 Nishigo Village, Japan. Geomophology 46, 117-128.

4. E. D. Kaplan (1996). Understanding GPS: Principles and Applications. London: Artech House Boston.

5. G. Xu (2007) GPS Theory, Algorithms and Application. Berlin: Springer.

6. K. Yedukondalu, A. D. Sarma and V. Satya Srinivas (2011) Estimation and mitigation of GPS multipath Interference using adaptive filtering. Progress In Electromagnetics Research M, (21), 133-148.

7. Yang Gao and Kongzhe Chen (2004) Performance analysis of precise point positioning using rea-time orbit and clock products. Journal of Global positioning,3(1-2), 95-100.

8. J. Kouba and P. Heroux (2001) GPS Precise Point Positioing Using IGS Orbit Products. GPS Solution $5(2), 12-28$

9. B. Hofmann-Wellenhof, H. Lichtenegger and J. Collins (2001) GPS theory and practice, fifth, revised edition.

10. Tomislav Kos, Ivan Markezic and Josip Pokrjcic (2010) Effects of multipath reception on GPS Positioning Performance. Proceedings ELMAR-2010 